\title{
A BACIA DE CAMPOS NO MESOCRETÁCEO: UMA CONTRIBUIÇÃO À PALEOCEANOGRAFIA DO ATLÂNTICO SUL PRIMITIVO
}

\author{
DIMAS DIAS-BRITO*
}

\begin{abstract}
This work integrates paleoecological research data obtained in Campos Basin and others areas of the Brazilian continental margins with those from different regions of the South Atlantic. A general model is proposed for the oceanographic evolution of the South Atlantic in the Mid-Cretaceous. Since the Early-Middle Albian, probably since the Aptian-Albian transition, the area extending from the equatorial region to the São Paulo Plateau-Walvis Ridge complex was simultaneously invaded by waters from the North (Tethys Sea) and South. The sea then formed constituted a long, continuous, and narrow epicontinental sea-way. Its waters were warm, physic-chemically unstable, and probably hypersaline. In the Late Albian, the South Proto-Atlantic had its salinity decreased to more normal marine values. It was after a significant anoxic event in Cenomanian-Turonian times that truly oceanic deposition phase started.
\end{abstract}

INTRODUÇÃo A origem e a evolução do Oceano Atlântico Sul têm sido investigadas por grande número de geocientistas na última década. Dados geofísicos, geoquímicos, sedimentológicos e paleobiológicos foram continuamente empregados, havendo significativo avanço no conhecimento geo-histórico desse oceano pela integração dessas informações. Entretanto, as fases iniciais da abertura do Atlântico Sul são ainda relativamente obscuras, e interpretações divergentes têm surgido em torno de variadas questões, tais como: quando houve a conexão Atlântico Sul-Atlântico Norte? Qual a profundidade das águas no Cretáceo "Médio"? As águas eram quentes ou frias? Estavam os continentes Sul-Americano e Africano em posição latitudinal muito diferente da ocupada por eles atualmente? Quais as características físicas e químicas das águas de fundo? Que tipo de fauna e flora pululava neste megaecossistema? Quais eram as condições climáticas?

Este trabalho objetiva abordar alguns desses aspectos, considerando-se os dados obtidos em várias bacias brasileiras, sobretudo a partir da Bacia de Campos, e aqueles provenientes de outras áreas fora da margem atlântica brasileira fornecidos pelo Deep Sea Drilling Project (DSDP). $\mathrm{O}$ enfoque é paleoecológico, sendo discutida a fase pré-oceânica do Atlântico Sul (Aptiano-Cenomaniano/ Turoniano); e uma breve síntese da fase rifte, anterior, é também apresentada.

\section{BREVE PANORÂMICA DA FASE RIFTE Durante o} Eocretáceo, fase Neocomiano-Aptiano, o Brasil, a África Ocidental e a África Central estiveram submetidos, de maneira geral, a um clima quente e árido/semi-árido; isto não só em função das condições climáticas planetárias então vigentes (clima quente e uniforme, Barron 1983) mas também porque esses territórios estavam a grandes distâncias dos oceanos Pacífico e Índico e, portanto, não recebiam os ventos úmidos das monções (Vakhrameev 1984). Este autor soviético, com base em estudos palinológicos, descreve a vegetação dessas áreas como constituída por arbustos esparsos de caráter xerofílico.

A disjunção dos continentes sul-americano e africano, iniciada há aproximadamente 125,5 Ma (Larson \& Ladd 1973 e Rabinowitz 1976, apud Barron et al. 1981), estava em pleno curso nesta época e os grábens formados ao longo do eixo de ruptura, sob a forma de paleoecossistemas lacustres, foram preenchidos por espessos depósitos sedimentares. As características bióticas e abióticas de tais lagos variaram geográfica e temporalmente. Corpos lacustres de água doce, como o Lago Recôncavo, com rica fauna de ostracodes, e de água salgada, como o Lago Lagoa Feia-Bacia de Campos (Rodrigues \& Takaki 1987, Carvalho et al. 1984), são exemplos desta variabilidade.

O ATLÂNTICO SUL NO APTIANO No Aptiano, o então nascente oceano caracterizava-se por ser fisiograficamente um estreito e alongado corpo d'água seccionado ao meio por uma cadeia vulcânica Walvis Ridge-São Paulo Ridge (Fig. 1). Tal feição, posicionando-se subperpendicularmente ao protomar, determinava a contemporaneidade de duas grandes bacias, aqui denominadas Atlântico Sul Setentrional e Atlântico Sul Meridional.

Elevações vulcânicas localizadas nas zonas de fraturas do Atlântico Equatorial (Mascle 1975 apud Kumar \& Gamboa 1979) impediam o contato livre do Atlântico Norte com os segmentos equatorial e setentrional do Atlântico.Sul, enquanto o segmento meridional recebia águas marinhas somente por uma tortuosa trilha ao longo da zona de fratura de Agulhas (Franchetau \& Le Pichon 1973 e Dingle \& Scrutton 1974 apud Natland 1978). Por sua vez, as bacias setentrional e meridional se conectavam de maneira apenas intermitente, o que ocorria quando pequenas quantidades de água passavam de sul para norte através de estreitos sills do complexo Walvis-Platô de São Paulo (Natland op. cit.).

O Atlântico Setentrional, nessa fase, pơr suas características fisiográficas, por estar submetido a um clima quente e

\footnotetext{
* Centro de Pesquisas da Petrobrás (Cenpes), Setor de Bioestratigrafia e Paleoecologia. Cidade Universitária, Quadra 7, Prédio 20, Ilha do Fundão, CEP 21910, Rio de Janeiro, RJ, Brasil
} 


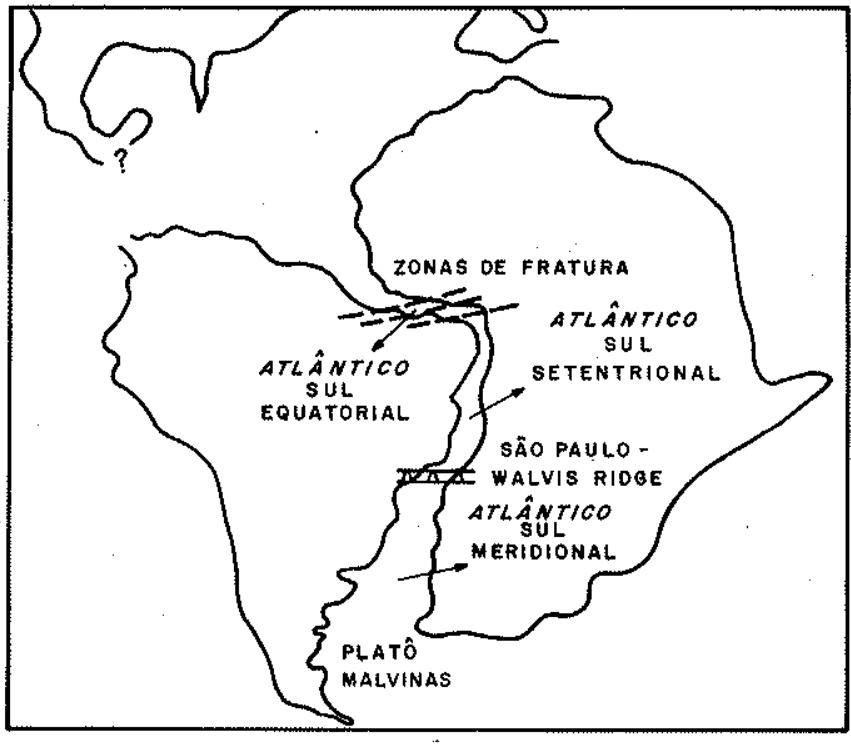

Figura 1 - Atlântico Sul no Aptiano

seco (Morgan 1978) e por receber somente reduzidos fluxos de água marinha, foi palco de acumulação de extensos depósitos evaporíticos. Tais corpos são registrados nas margens leste brasileira e oeste africana; recentemente, foi também constatada a ocorrência de um corpo evaporítico na margem equatorial do Brasil, na Bacia do Ceará.

O Atlântico Meridional, por seu turno, constitura-se em ambiente marinho euxínico, onde se depositavam sedimentos sapropélicos sob uma massa de água que durante todo o Aptiano manteve-se anóxica (Natland 1978).

\section{A BACIA DE CAMPOS NO MESOCRETACEO: A FASE} PRÉ-OCEÁNICA A coluna litológica da Bacia de Campos engloba sedimentos depositados em quatro fases distintas: lacustre; evaporítica s.s:; pré-oceânica; e oceânica.

Aos depósitos lacustres sobrepuseram-se espessos pacotes de sais gerados em meio hiper-restrito, o que assinalou o início da presença marinha na área.

O estágio carbonático subseqüente, de idade albiana, consolidou a instalação da sedimentação marinha e constituiu a transição de um sistema hiper-restrito para um sistema oceânico; revestiu-se, portanto, de características muito peculiares. Iniciado pela deposição de calcarenitos oncolíticos/oolíticos com os níveis inferiores às vezes dolomitizados, evoluiu para calcilutitos que paulatinamente gradaram para margas e folhellhos pós-albianos. Esta unidade litológica constitui a chamada Formação Macaé, e a investigação de sua evolução paleoceanográfica (Dias-Brito 1982) resultou no conhecimento dos parâme. tros paleoecológicos dominantes neste segmento do Atlântico Sul durante o Mesocretáceo.

Abaixo são apresentados os fatores bióticos e abióticos postulados para o paleoecossistema estudado e discutidas as informaçōes disponíveis na literatura a respeito da paleoceanografia do Atlântico Sul primitivo.

Paleobatimetria $O$ pacote albiano depositado na Bacia de Campos contém uma paleobiota essencialmente de ambiente nerítico (Dias-Brito 1982, Azevedo et al. 1987). Em outras bacias brasileiras também só são registrados foraminiferos de águas rasas, o que é discutido em detalhe em Koutsoukos \& Dias-Brito (1987).

Em relação ao intervalo Cenomaniano-Turoniano, a pro- fundidade deposicional oscilou entre 200 e $300 \mathrm{~m}$ (Azevedo et al. 1987).

A constatação paleobatimétrica mencionada é semelhante à referente aos intervalos de carbonatos finos albianos da Bacia de Angola e Walvis Ridge, perfurados pelo DSDP, Leg 40, sites 364 e 363, respectivamente. Caron (1978), ao analisar o conteúdo de foraminiferos planctônicos, identifica a presença de episódios neríticos. nas referidas seções. Scheibnerová (1978) também menciona que os foraminíferos bentônicos do Aptiano e Albiano representam associações de águas rasas que se correlacionam diretamente com associaç̋̃es nearshore do leste da África do Sul, do sul da India peninsular e da Grande Bacia Australiana. Por outro lado, Wiedmann \& Neugebauer (1978), ao estudarem os amonites do Cretáceo Inferior do site 364 , assinalam que esses bioelementos estão, no Albiano, relacionados a ambiente nerítico interno ou epicontinental. Melguen (1978), ao propor uma evolução paleobatimétrica para ambos os sites, chama a atenção para o fato de que estes foram extremamente rasos durante o Aptiano/Albiano.

Recentemente, a partir dos dados obtidos pelo DSDP, Legs 26, 36 e 40, Scheibnerová (1981) fez uma síntese comparativa dos foraminíferos bênticos albo-cenomanianos encontrados no Atlântico Sul. Este estudo permitiu à pesquisadora postular que, nesse tempo, este oceano era, provavelmente, umà bacia rasa com profundidades em torno de $100 \mathrm{~m}$, não excedendo, em locais mais profundos, a 300 e $400 \mathrm{~m}$.

Todos esses dados paleobiológicos estão em flagrante desacordo com inferências paleobatimétricas obtidas por outros métodos geotectônicos, reconstrução de CCD, cujas estimativas sugerem valores de profundidade para o Atlântico Sul albiano maiores que $3.000 \mathrm{~m}$ (Sclater \& McKenzie 1973, Van Andel et al. 1977, Melguen et al. 1978, discutidos em maior detalhe em Koutsoukos \& Dias-Brito 1987).

Paleoclima e paleotemperatura das águas A sedimentação carbonática da Bacia de Campos deu-se em meio marinho de águas tropicais, sob um clima quente e seco (Dias-Brito 1982). Tal dedução baseia-se nas características dos calcarenitos da base da seqüência: apresentam grãos com envelopes micríticos, oblitos e pelóides, que, de acordo com Leonard et al. (1981), só se formam em águas quentes. Também os carbonatos finos, sobrejacentes aos calcarenitos, são portadores de foraminíferos cujas características morfofuncionais são típicas de faixas epipelágicas subtropicais-tropicais. E o caso de Favusella washitensis. Também a ocorrência do nanoplâncton calcário Nannoconus truitti, que, segundo Wiedmann \& Neugebauer (1978), tem claras relaçōes com formas tetianas, é indicadora de que águas tropicais preenchiam a bacia albiana. Isso também é sugerido pela efetiva presença de Calcisphaerulidae nos sedimentos; tais elementos esferiformes são caracterf́sticos dos depósitos tetianos do Albiano.

A partir das investigaçóes feitas no lado africano, Morgan (1978) e Siesser (1978) também postularam um clima tropical, possivelmente quente e semi-árido para o Albiano. Moullade \& Guérin (1982) assinalam que os foraminiferos Heterohelix washitensis, Ticinella primula, Gavelinella flandrini e $G$. intermedia, encontrados no Atlântico Sul Setentrional - sites 356,363 e 364 DSDP -, são formas abundantes da província "Mesogeenne" (Tethys + Atlântico Central) e que não poderiam viver em aguas relativa. mente frias.

Entretanto, Scheibnerová (1978) e Caron (1978) advo- 
gam que as áreas da Bacia de Angola e Walvis Ridge teriam estado sob a influência de águas austrais frias naquele tempo. Tal hipótese também é apresentada por Premoli Silva \& Boersma (1977) em relação às águas albianas que cobriam o Platô de São Paulo. Todos esses autores, especialistas em foraminíferos, sustentam suas interpretaçôes na idéia de que tănto os planctônicos como os bentônicos apresentam sinais de fauna austral. Esses sinais são, sobretudo, o domínio exclusivo de elementos planctônicos globigeriniformes e as reduzidas dimensōes dos espécimes.

A forte divergència acima apresentada pode desaparecer, no entanto, se a interpretação para os "sinais de fauna austral" for contestada. Outros fatores, que não as baixas temperaturas das águas, devem ter afetado o crescimento dos indivíduos e determinado a ausência absoluta de foraminíferos planctônicos quilhados nas águas albianas do Atlân* tico Sul.

Baixos valores batimétricos constituem barreiras naturais que impossibilitam a presença de foraminíferos planctônicos quilhados em águas neríticas (Douglas \& Rankin 1969, Frerichs 1971, Douglas \& Savin 1978). Tais organismos, representados no Albiano por Praeglobotruncana, Rotalipora e Planomalina (Haig 1979), foram segregados a niveis mais profundos pelo menos desde este intervalo de tempo (Douglas \& Savin op. cit.). Ora, ao levar-se em conta que os depósitos albianos não se acumularam em ambiente profundo, parece claro o motivo pelo qual não se encontram planctônicos quilhados nos sedimentos. Outrossim, os globigeriniformes, que, quando exclusivos em águas tropicais, indicam áreas neríticas, tiveram seu nanismo ligado às con" diçōes relativamente adversas na coluna de água. As faixas pelágicas, em função da restrição marinha então vigente (adiante será discutida), constituíam um universo físico-químico particular e instável, que exercia forte controle sobre o crescimento dos espécimes.

Ao se considerar que os bioelementos (foraminiferos, calcisferulídios e nanofósseis calcários) do Albiano da margem atlântica brasileira constituem um conjunto relativamente uniforme em todas as bacias, entende-se que as águas dos megaecossistemas Atlântico Setentrional e Atlântico Equatorial eram tropicais e que um clima quente e seco dominara essas áreas; suas posiçôes latitudinais eram semelhantes às presentes. Tais condições climáticas também são postuladas por Vakhrameev (1984) ao discutir as características da flora dessas regiōes no Albiano.

A partir da transição Neoalbiano-Cenomaniano, o clima na Bacia de Campos alterou-se radicalmente para quente e úmido, determinando a interrupção da sedimentação carbonática e o início de uma deposição de margas. No intervalo Cenomaniano-Turoniano, folhelhos se acumularam em condiçóes anóxicas. A referida mudança climática é advogada também por Vakhrameev (1984), que assinala a diminuição, no Cenomaniano, do pólen Classopollis produzido por coníferas xerofílicas que dominaram amplamente ao longo de todo o Eocretáceo quente e seco; no Turoniano, tal pólen praticamente desapareceu, tempo que o referido palinológo admite como início dos protótipos da floresta úmida tropical no cinturão equatorial da África e da América do Sul. Viviers \& Regali (1987) também acentuam que. na Bacia Potiguar (Atlântico Equatorial), durante a transição Cenomaniano-Turoniano, ocorreu uma grande mudança florística em decorrência do encerramento do ciclo climático quente e árido. A causa da alteração climática - quente e seca para quente e úmida - deve ter sido provocada pelo aumento no volume de água do Atlântico
Sul (incluindo todos os seus segmentos) em decorrência de seu crescente contato com massas de água dos oceanos do sul e do norte; isto derivava de um gradativo afastamento dos continentes e de um progressivo incremento na subsidência das grandes bacias.

A aparentemente precoce modificação climática na área sudeste brasileira (transição Neoalbiano-Cenomaniano) em relação à mencionada transição Cenomaniano-Turoniano pode sugerir que:

a. ou o clima no sudeste mudou alguns milhōes de anos antes;

b. ou a flora reagiu de maneira relativamente "lenta" às mudanças, o que não se deu em termos de alteração litológica. Este "retardo florístico" talvez tenha sido detectado por Vakhrameev (op. cit.) ao assinalar a referida "diminuição, no Cenomaniano, do pólen Classopollis".

Restriçẫo ambiental $\mathrm{O}$ acúmulo de sedimentos carbonáticos e argilosos na Bacia de Campos durante o Mesocretáceo deu-se em ambiente restrito (Dias-Brito 1982). Tal afirmativa baseia-se na baixa diversidade apresentada pelos foraminiferos bentônicos e planctônicos presentes na seqüência Macaé e na escassez pronunciada desses bioelementos, que são também relativamente pequenos.

Durante a fase carbonática, a bacia, com balanço negativo de águas, apresentava fundos bem oxigenados, fato evidenciado pela presença de bioturbaçōes, pelas cores claras e pelo baixo conteúdo de carbono orgânico que caracterizam os carbonatos finos albianos. Mares rasos com pisos ventilados em áreas tropicais constituem palco de proliferação das comunidades bênticas. O Mar Macaé, raso, oxigenado e tropical, no entanto, teve reduzida população de fundo. Tal paradoxo possibilitou a conclusão que havia condiçôes anômalas, provavelmente hipersalinidade das águas, no fundo marinho. Esta hipersalinidade também afetaria as faixas pelágicas superiores, acarretando problemas ao metabolismo de crescimento e reprodução dos foraminíferos. Aí estaria a causa do nanismo, da atipicidade e do empobrecimento apresentado pelos foraminíferos.

Natland (1978) também considera que na Bacia de Angola as fácies carbonáticas albianas depositaram-se em meio restrito e hipersalino. A hipersalinidade é postulada com base na ocorrência de calcilutitos afossilíferos, na ocorrência de cristais de dolomita excepcionalmente ricos em sódio, na presença de folhelhos contendo a associação dos minerais autigênicos ilita-filipsita-caulinita e no fato de que os carbonatos acumularam-se logo após os evaporitos. Este autor atribui, ainda, condiçōes anóxicas à bacia angolana nessa fase. Processos de anoxia verificaram-se na Bacia de Campos na transição da sedimentação carbonática para a argilosa ocorrida no Albiano Superior e, sobretudo, quando da deposição do espesso pacote de folhelhos pós-albianos. Esta fase argilosa deu-se em bacia com balanço positivo de águas, sendo que o acúmulo de sedimentos deu-se sob a. forma de estratos finamente laminados com cristais de pirita e raros foraminfferos bentônicos. A coluna de água também parece não ter sido propícia à vida de bioelementos calcários, já que o plâncton preservado é raro. $O$ aporte de terrígenos à bacia teria sido fator decisivo na interrupção da sedimentação carbonática. Esse evento argiloso corresponde, na realidade, a um dos momentos anóxicos que marcaram o Atlântico Sul no Mesocretáceo (Schlanger \& Jenkyns 1976, Ryan \& Cita 1977, Arthur \& Natland. 1979, Arthur \& Schlanger 1979, Tissot et al. 1980, Brumsack 1980). 
A CONEXÃO DO ATLÁNTICO NORTE E ATLÅNTICO SUL NO ALBIANO A ligação dos oceanos Atlântico Norte e Sul tem sido motivo de polêmica, principalmente no que concerne ao tempo que este fato ocorreu (Tab, 1).

No terreno paleobiogeográfico, especialistas em amonóides e foraminiferos compóem o grupo que mais tem apresentado dados e interpretações a respeito da conexão Atlântico Sul-Atlântico Norte.

Premoli Silva \& Boersma (1977) e Caron (1978) argumentam que o contato das águas realizou-se durante o Cenomaniano. Esta hipótese está sustentada pela idéia de que, antes deste tempo, o Attântico Sul era habitado somente por foraminíferos planctônicos de águas frias. Tal idéia é contestada neste trabalho. Também Scheibnerová (1981) sustenta que 0 contato Atlântico Sul-Atlântico Norte aconteceu algum tempo depois do Cenomaniano.

Wiedmann \& Neugebauer (1978), com base em estudos de amonites, inferem que a conexão Atlântico Sul-Atlântico Norte talvez tenha acontecido já no Neoaptiano, mas consideram que a história comum dos dois oceanos tenha começado no Mesoalbiano (Fig. 2). Eles sustentam que a pretensa barreira que ligava o Nordeste do Brasil à Nigéria

Tabela 1 - Proposiçóes de vários pesquisadores acerca do tempo que se iniciou a conexão dos oceanos Atlântico Sul e Atlântico Norte

\begin{tabular}{|l|l|l|}
\hline Ano & \multicolumn{1}{|c|}{ Tempo de conexã́o } & \multicolumn{1}{|c|}{ Autor } \\
\hline 1961 & Pós-Turoniano & Beurlen \\
1969 & Pós-Turoniano & Maak \\
1972 & Turoniano & Reyment \& Tait \\
1973 & Cretáceo terminal & Scheibnerová \\
1973 & Aptiano-Albiano & Nairn \& Stehli \\
1974 & Neocenomaniano & Berggren \& Hollister \\
1974 & Neoalbiano & Carvalho et al. \\
1975 & Neoalbiano & Kennedy \& Cooper \\
1976 & A partir do Turoniano & Bengtson \& Tait \\
1977 & A partir do Turoniano & Reyment \& Mörner \\
1977 & Neoalbiano-Cenomaniano & Sclater et al. \\
1977 & Neoalbiano-Cenomaniano & Sliter \\
1978 & Neoaptiano-Mesoalbiano & Wiedmann \& Neugebauex \\
1978 & Neoalbiano-Cenomaniano & Förster \\
1978 & Pós-Cenomaniano & Scheibnerová \\
1978 & Cenomaniano & Premoli Silva \& Boersma \\
1978 & Cenomaniano & Caron \\
1978 & A partir do Turoniano & Berggren \\
1979 & Neoalbiano-Cenomaniano & Förster \& Scholz \\
1981 & Albiano & Tibana \& Terra \\
1982 & Mesoalbiano & Moulade \& Guérin \\
1982 & Pós-Maastrichtiano & Rand \& Mabesoone \\
1983 & Eo-Mesoalbiano & Dias-Brito \\
1984 & Cenomaniano ou Eoturoniano & Vakhrameev \\
\hline & & \\
\hline
\end{tabular}

(Beurlen 1961, 1974, Maack 1969, Reyment \& Tait 1972 apud Wiedmann \& Neugebauer op. cit.) não é justificada, já que faunas européias (amonites, inoceramídios e nanoflora) invadiram o Atlântico Sul no Albiano (Fig. 2). Tais resultados estão de acordo com os obtidos pelos amonitólogos Kennedy \& Cooper (1975), os quais fundamentados em dados paleobiogeográficos, postularam a submergência da "barreira albiana-turoniana" no Neoalbiano.

Moullade \& Guérin (1982), num excelente artigo especidância com Wiedmann \& Neugebauer (1978), que o contato já ocorria no Mesoalbiano por meio de um sill colocado a uma profunidade de 150 a $200 \mathrm{~m}$. Aqueles autores relacionam cerca de 95 espécies de foraminíferos que, durante o Mesoalbiano, são comuns aos oceanos Atlântico Sul Setentrional, Atlântico Sul Equatorial, Átlântico Central e Tethys:

Na margem atlântica brasileira, em suas diversas bacias do lestè e da zona equatorial, foraminiferos planctônicos, igualmente de origem tetiana, também são freqüentemente encontrados ao longo do Albiano: Favusella spp., Globigerinelloides barri, G. ex gr. casey, G. gyroidinaeformis, Hedbergella ex gr. delrioensis, $H$. aff. gorbachikae, $H$. luterbacheri, $H$. aff. maslakovae, $H$. aff. trocoidea, Heterohelix moremani, Ticinella bejaouensis, $T$. aff. primula, T. roberti e Whiteinella spp. As espécies de foraminiferos bentônicos tetianos podem ser conhecidas em Koutsoukos \& Dias-Brito (1987).

Dias-Brito (1985a, 1985b), ao investigar a presença de calcisferulídios em carbonatos finos do Albiano da Bacia de Campos e da Bacia Potiguar, concluiu que, desde o

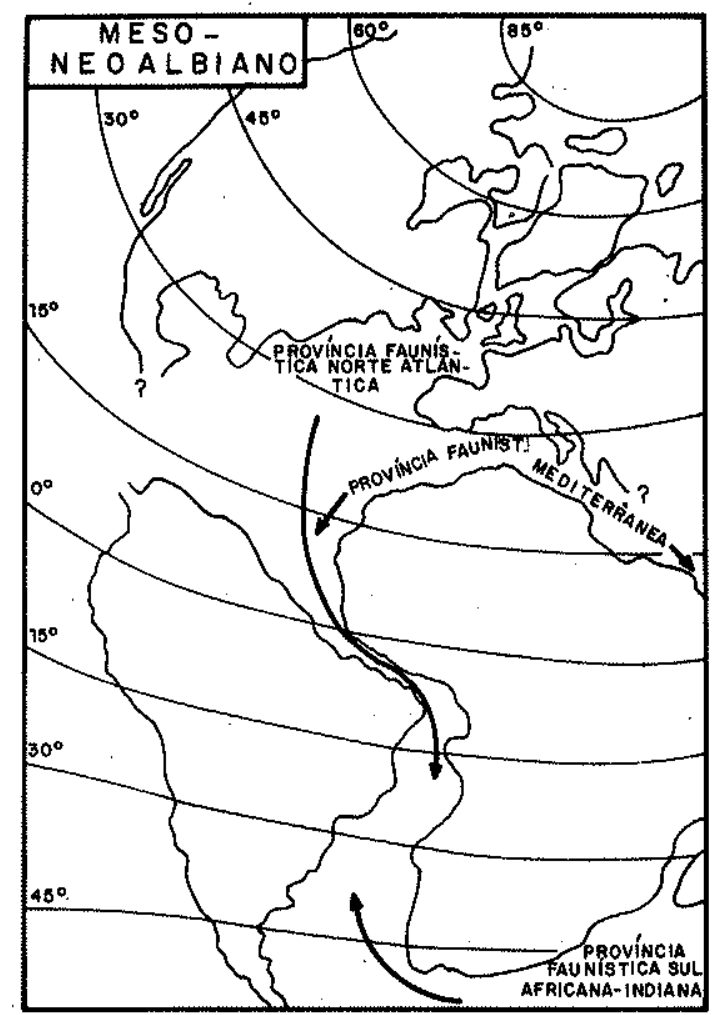

Figura 2 - Conexão do Atlântico Sul-Atlântico Norte. Evidência faunistica (Wiedmann \& Neugebauer 1978)

Eo-Mesoalbiano, os oceanos Atlântico Equatorial eiSetentrional já estavam ligados e, por outro lado, já conectados com o Atlântico Norte. No Neoalbiano ocorreu uma intensificação no contato desses dois oceanos, dessalinizando e normalizando as águas marinhas até então hipersalinas.

Todos os dados acima demonstram o forte caráter tetiano das associações fossillferas albianas do Atlântico Sul Equatorial e Atlântico Sul Setentrional, opondo-se frontalmente à tese do "caráter austral" da paleobiota defendida por Bolli (1978).

A possibilidade de que o Atlântico Sul e o Atlântico Norte já estivessem ligados desde o Aptiano terminal não pode ser descartada. Na Bacia Sergipe-Alagoas, o topo do Andar Alagoas (andar local), que foi definido como Aptia- 
no terminal baseado em amonóides, contém Nannoconus truitti e Favusella washitensis (espécies tetianas); amonóides de distribuição quase que mundial (segundo Beurlen, apud Schaller 1969) também ocorrem neste nível. Por outro lado, Viviers (apud Della Fávera et al. 1984) descreve na Bacia do Ceará, Atlântico Sul Equatorial, uma associação de foraminíferos planctônicos com afinidades aptianas e tetianas, incluindo Hedbergella aff. bollii e $H$. aff. roblesae. Estes fatos levam a pensar na possibilidade de que a ruptura completa dos continentes sul-americano e africano teria ocorrido imediatamente após a fase evaporítica; o desaparecimento de barreiras teria permitido que águas oriundas do Atlântico Norte e Atlântico Sul Meridional invadissem as áreas dos oceanos Atlântico Setentrional e Atlântico Equatorial, encerrando o ciclo de deposição salífera e paulatinamente incrementando o homogeneizando a lâmina de água desses oceanos. A manifestação mais efetiva desse contato de águas verificou-se mais tarde, talvez no pós-Turoniano, quando correntes de fundo teriam erodido estratos depositados provavelmente de maneira contínua durante o Cenomaniano e Turoniano (Morgan 1978). Esses possíveis processos erosivos, causadores de cânions e hiatos, atuaram tanto na costa africana quanto em grande parte da margem continental brasileira e refletiram o processo de desestabilização do Atlântico Sul primitivo (Fig.3).

CONCLUSÕES Dados paleoecológicos permitem reconstituir hipoteticamente o cenário paleoambiental da Bacia de Campos no Mesocretáceo.

Durante o Eoalbiano e Mesoalbiano, o primitivo Atlântico Sul prosseguia em sua expansão inicial, principiada nesta área em tempos aptianos sob condições evaporíticas. Suas águas eram neríticas rasas, oxigenadas, pobres em nutrientes e hipersalinas; sua biota era escassa e pouco diversificada. No Neoalbiano, o ambiente tornou-se mais prolífico e menos salgado em decorrência de um importante incremento no contato entre os oceanos Atlântico Sul e Norte. Um clima cíclico quente-seco/quente-úmido estabeleceu-se, sendo que, ao final deste intervalo de tempo, as alternâncias climáticas tornaram-se mais proeminentes, controlando a sedimentação rítmica de calcilutitos e margas. Durante essa fase ocorreram ciclos anóxicos efêmeros, precedendo significativo evento de anoxia ocorrido no intervalo Cenomaniano-Turoniano.

Como modelo geral para a história evolutiva do Atlântico Sul, é proposto que a partir do Eo-Mesoalbiano, talvez desde a transição Aptiano-Albiano, a área que abrange desde a região equatorial até o sistema Platô de São Paulo-Walvis amplo, com salinidade mais baixa que a registrada na etapa precedente, sem contudo mostrar caráter "mar normal". Ao

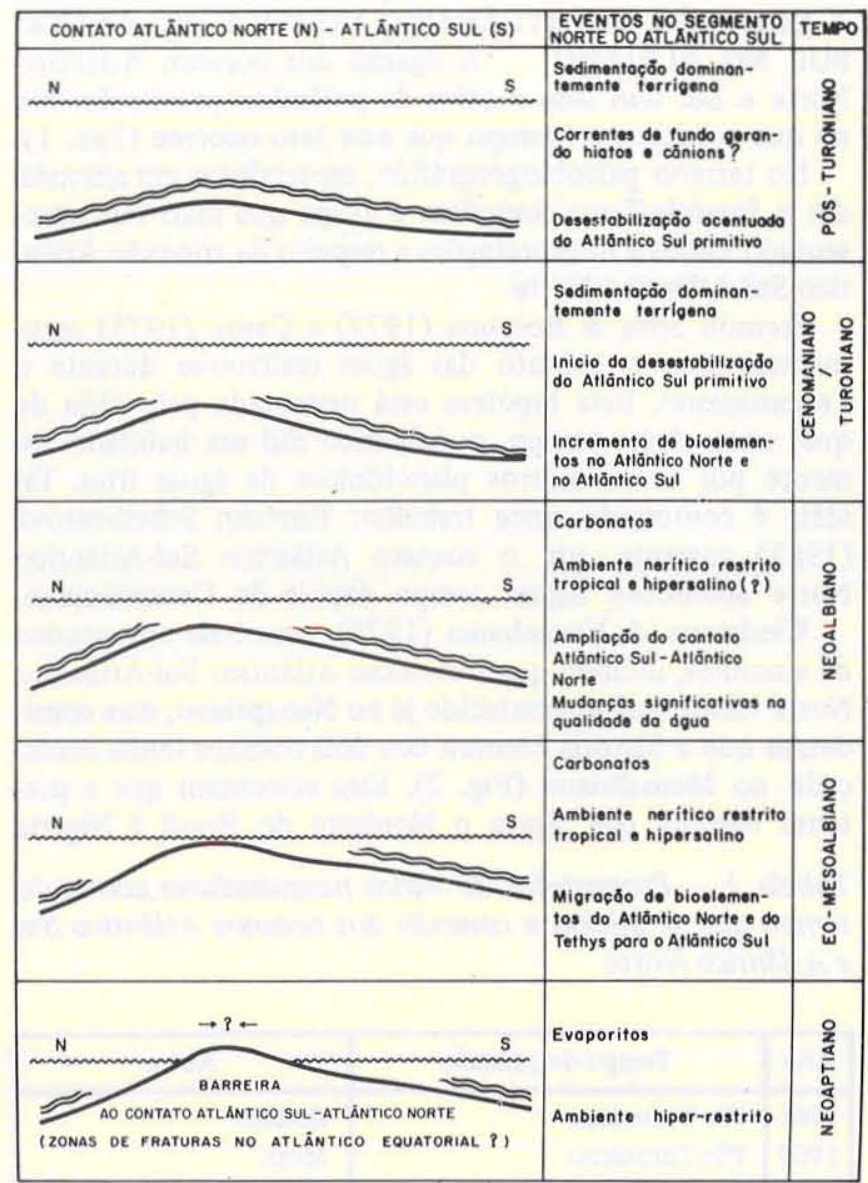

Figura 3 - Evolução hipotética da conexão dos oceanos Atlântico Sul-Atlântico Norte

ingressar no tempo Cenomaniano-Turoniano, o Atlântico Sul experimentou episódios de anoxia, após o que iniciou sua fase oceânica propriamente dita.

Ridge tenha sido simultaneamente invadida por águas oriundas do norte e do sul. A massa de água então formada constituiu-se em um longo, contínuo e estreito mar epicontinental, com águas quentes, físico-quimicamente instáveis e provavelmente hipersalinas. No Neoalbiano, o corpo marinho, por já estar ligado de modo mais efetivo com as massas oceânicas do norte e do sul, revelou-se mais profundo e

Agradecimentos $\mathrm{O}$ autor expressa sua gratidão à gerência da Petróleo Brasileiro S.A. por permitir a divulgação destes resultados e propiciar sua apresentação no I Simpósio sobre a Evolução do Atlântico Sul.

\section{REFERÊNCIAS BIBLIOGRÁFICAS}

ARTHUR, M.A. \& NATLAND, J.H. - 1979 - Carbonaceous sediments in the North and South Atlantic: the role of salinity in stable stratigrafication of early cretaceous basins. In: TALWANI, M.; WAY, W.; RYAN, W.B.F., eds. Deep drilling results in the Atlantic ocean: continental margins and paleoenvironment. Washington, American Geophysical Union, p. 375-401.

ARTHUR, M.A. \& SCHLANGER, S.O. - 1979 - Cretaceuos oceanic anoxic events as causal factors in development of reef reservoired giant oil fields. AAPG Bull., 63(6):870-885.

AZEVEDO, R.L.M. de; GOMIDE, J.; VIVIERS, M.C. - 1987 Geo-história da Bacia de Campos-Brasil: do Albiano ao Maastrichtiano. Rev. Bras. Geoc., 17(2):139-146.

BARRON, E.J. - 1983 - A warm, equable Cretaceous: the nature of the problem. Earth-Science Rev., 19:305-338.

BARRON, E.J.; HARRISON, C.G.A.; SLOAN II, J.L.; HAY, W. 1981 - Paleogeography, 180 million years ago to the present.
Eclogae geol. Helv., 74(2):443-470.

BERGREEN, W.A. \& HOLLISTER, C.D. - 1974 - Paleogeography, paleobiogeography and the history of circulation of the Atlantic Ocean. Proc. Symp. Geol. Hist. Oceans, Soc. Econ. Pal. Mineral, Mem. 20:126-186.

BOLLI, H.M. - 1978 - Cretaceous and Paleogene calcisphaerulidae from DSDP Leg 40, southeastern Atlantic, In: BOLLI, H.M., RYAN, W.E.F., et al. eds. Initial Reports of the Deep Sea Drilling Project. Washington, U.S. Government Printing Office v. 40 , p. $819-837$.

BRUMSACK, H.J. - 1980 - Geochemistry of Cretaceous black shales from the Atlantic Ocean (DSDP Legs 11, 14, 36 and 41). Chemical Geology, 31:1-25.

CARON, M. - 1978 - Cretaceous planktonic foraminifers from DSDP leg 40, Southeastern Atlantic Ocean. In: BOLLI, H.M.; RYAN, W.E.F. et al. Init. Repts. DSDP. Washington. (U.S. 
Govt. Printing Office), 40:651-678.

CARVALHO, R.S.; SIMOEES, I.A.; TIBANA, P.; LEITE, D.C. 1974 - Evaporitos de Sergipe, Projeto evaporitos. Rio de Janeiro. Petrobrás (Rel. Int.).

CARVALHO, M.D.; MONTEIRO, M.; PIMENTEL, A.M.; REHIM, H.A.A.A. - 1984 - Microfácies, diagênese e petrofisica das coquinas da Formação Lagoa Feia em Badejo, Linguado $e$ Dimpo, Bacia de Campos. Petrobrás (Rel. int.).

DELLA FĀVERA, J.C.; MEDEIROS, R.A.; APPI, C.J.; BEURLEN, G.; VIVIERS, M.C.; HASHIMOTO, A.T. - 1984 - Análise estratigráfica do Andar Alagoas na Bacia do Ceará. Petrobrás (Rel. int.).

DIAS-BRITO, D. - 1982 - Evolução paleoecológica da Bacia de Campos durante a deposição dos calcilutitos, margas e follhelhos da Formação Macaé (Albiano e Cenomaniano?). Bol. Téc. Petrobrás, 25(2):11-24.

DIAS-BRITO, D. - 1985a - Calcisphaerulidae do Albiano da Bacia de Campos, Rio de Janeiro, Brasil. Investigações taxonômicas: biocronoestratigráficas e paleoambientais. In: Brasil, MME, DNPM. Col. Trab. Paleont. Brasília. (Geologia 27; Paleontologia e Estratigrafia 2:295-306).

DIAS-BRITO, D. - 1985b - Calcisphaerulidae e microfósseis associados da Formação Ponta do Mel-Bacia Potiguar, Brasil: considerações paleoecológicas e biocronoestratigráficas. In: Brasil, MME, DNPM. Col. Trab. Paleont. Brasília. (Geologia 27, Paleontologia e Estratigrafia 2:307-314).

DOUGLAS, R.G. \& SAVIN, S.M. - 1978 - Oxigen isotopic evidence for the depth stratification of Tertiary and Cretaceous planktic foraminifera. Marine micropaleontology, 3:175-196.

FORSTER, R, - 1978 - Evidences for an open seaway between northern and southern proto-Atlantic in Albian times. Nature, 272(5649):158-159.

FORSTER, R. \& SCHOLZ, G. - 1979 - Salazicerus nigerianun n. sp. from Southeast Nigeria: Faunal evidence for an open seaway between the northern and southern Atlantic in Late Albian times. $N$. Jb. Geol. Palaont, Mh, H. 2:109-119.

FRERICHS, W.E. - 1971 - Planktonic foraminifera in sediments of the Andaman Sea. J. Foraminiferal Research, 1:1-14.

HAIG, D.W. - 1979 - Global distribution patterns for mid-Cretaceous foraminiferids. J. Foraminiferal Research, 9(1):29-40.

KENNEDY, W.J. \& COOPER, M. - 1975 - Cretaceous ammonite distributions and the opening of the South Atlantic. J. Geol. Soc. London, 131:283-288.

KOUTSOUKOS \& DIAS-BRITO, D. - 1987 - Paleobatimetria da margem continental do Brasil durante o Albiano. Rev. Bras. Geoc., 17(2): 86-91.

KUMAR, N. \& GAMBOA, L.A.P. - 1979 - Evolution of the São Paulo Plateau (southeastern Brazilian margin) and implications for the early history of the South Atlantic. Geol. Soc. of Am. Bull., Part I, 90:281-293.

LEONARD, J.E.; CAMERON, B.; PILKEY, O.H.; FRIEDMAN, G.M. - 1981 - Evaluation of cold-water carbonates as a possible paleoclimate indicator. Sedimentary Geology, 28:1-28.

MELGUEN, M. - 1978 - Facies evolution, carbonate dissolution cicles in sediments from the Eastern South Atlantic (DSDP Leg 40) since the Early Cretaceous. In: BOLLI, H.M.; RYAN, W.B.F. et al. Init. Repts. DSDP. Washington, (U.S. Govt. Printing Office), 40:981-1024.

MELGUEN, M. et al. - 1978 - Paléoenvironnement de l'Atlantique Sud. Bull. Soc. Geol. France, 7, t. XX(4):471-489.

MORGAN, R. - 1978 - Albian to Cenomanian palynology of Site 364, Angola Basin. In: BOLLI, H.M.; RYAN, W.B.F. et al., Init. Repts. DSDP, Washington (U.S. Govt. Printing Office), 40:915-951.

MOULLADE \& GUERRIN - 1982 - Le problème des relations l'Atlantique Sud et de l'Atlantique Central au Cretácé moyen: nouvelles données microfauniques d'après de forages D.S.D.P. Bull. Soc. Geol., 7, E. XXIV(3):511-517.

NAIRN, A.E.M. \& STEHLI, F.G. - 1973 - A model for the South Atlantic. In: NAIRN, A.E.M. \& STEHLI, F.G. (eds.) The Ocean Basins and Margins.s. 1., p. 1-21, (v. 1, The South Atlantic).
NATLAND, J.H. - 1978 - Composition, provenance, and diagenesis of Cretaceous clastic sediments drilled on the Atlantic continental rise of Southern Africa, DSDP site 361 - Implications for the early circulation of the South Atlantic. In: BOLLI, H.M.; RYAN, W.B.F. et al. Init. Repts. DSDP Washington (U.S. Govt. Printing Office), 40:1025-1061.

PREMOLI SILVA, I. \& BOOERSMA, A. - 1977 - Cretaceous planktonic foraminifers-DSDP Leg 39 (South Atlantic). In: PERCH-NIELSEN, K. \& SUPKO, P., Init. Repts. DSDP, Washington (U.S. Govt. Printing Office), 40:1025-1061.

RAND, M.H. \& MABESOONE, J.M. - 1982 - Northeastern Brazil and the final separation of South America and Africa. Palaeog., Palaeoclimat., Palaeoecol., 38:163-183.

REYMENT R.A. \& MORNER, N.A. - 1977 - Cretaceous transgressions exemplified by the South Atlantic. Palaeont. Soc. Japan, p. 247-261. (Spec. paper $n .{ }^{\circ} 21$ ).

RODRIGUES, R. \& TAKAKI, T. - 1987 - O Cretáceo Inferior nas bacias sedimentares da costa sudeste do Brasil. Análise isotópica e suas implicações paleoambientais. Rev. Bras. Geoc., 17(2):177-179.

RYAN, W.B.F. \& CITA, M.B. - 1977 - Ignorance concerning episodes ocean-wide stagnation. Marine Geology, 23:197-215.

SCHEIBNEVORÁ, V. - 1978 - Aptian and Albian benthic foraminifers of Leg 40, sites 363 and 364, Southern Atlantic. In: BOLLI, H.M., RYAN, W.B.F., et al., Init. Repts. DSDP, Washington (U.S. Govt. Printing Office), 40:741-757.

SCHEIBNEVORÅ, V. - 1981 - Paleogeographical implication of cretaceous benthic foraminifers recovered by the Deep Sea Drilling Project in the Wostern South Atlantic Ocean. Cretaceous Research, 2:1-18.

SCHALLER, H. - 1969 - Revisão estratigráfica da Bacia de Sergipe/Alagoas. Bol. Téc. Petrobrás, 12(1):21-86.

SCHLANGER, S.G. \& JENKYNS, H.C. - 1976 - Cretaceous oceanic anoxic events, causes and consequences. Geologia Mijnbouw, 55:179-198.

SCLATER, J.G. \& MCKENZIE, D.P. - 1973 - Paleobathymetry of the South Atlantic. Geological Society of America Bull., 84(10):3203-3216.

SCLATER, J.G.; HELLINGER, S.; TAPSCOTT, C. -.1977 - The paleobathymetry of the Atlantic Ocean from the Jurassic to the Present. J. Geol., 85:509-552.

SIESSER, W.G. - 1978 - Leg 40 results in relation to continental shelf and onshore geology. In: BOLLI, H.M.; RYAN, W.E.F., et al. eds. Initial Reports of the Deep Sea Drilling Project. Washington, U.S. Government Printing Office. v. 40, p. 965-979.

TIBANA, P. \& TERRA, G.S. - 1981 - Seqüências carbonáticas do Cretáceo na Bacia Potiguar. Bol. Téc. Petrobrás, 24(3):174-183.

TISSOT, B.; DEMAISON, G.; MASSON, P.; DELTEIL; J.R.; KOMBAZ, A. - 1980 - Paleoenvironmental and petroleum potential of middle cretaceous black shales in Atlantic basin. AAPG Bull., 64(12):2051-63.

VAKHRAMEEV, V.A. - 1984 - O clima do Cinturão Tropical da África e América do Sul no Cretáceo Inferior. Dokl., Akad. Nauk., SSSR, 274(4):894-897 (em russo).

VAN ANDEL, T.H.; THIEDE, J.; SCLATER, J.G.; HAY, W.W. 1977 - Depositional history and paleooceanography of the South Atlantic Ocean during the last 125 million years. $J$. Geol., 85(6):651-698.

VIVIERS, M.C. \& REGALI, M.S.P. - 1987 - Estudo paleoambiental preliminar no Cretáceo da Bacia Potiguar. Rev. Bras. Geoc. 17(2):123-130.

WIEDMANN, J. \& NEUGEBAUER, J. - 1978 - Lower Cretaceous Ammonites from the South Atlantic Leg 40 (DSDP), their stratigraphic value and sedimentological, properties. In: Init. Repts. DSDP, suppl., Washington, (U.S. Govt. Print Office), 40:709-734.

MANUSCRITO 382 Recebido em 01 de setembro de 1986 Revisão aceita em 30 de março de 1987 